\title{
Modeling and Identification of Spacecraft Systems Using Adaptive Neuro Fuzzy Inference Systems (ANFIS)
}

\author{
Tharwat O. S. Hanafy ${ }^{1,2}$, Mosleh M. Al-Harthi ${ }^{1}$, Nadjim MERABTINE ${ }^{1}$ \\ ${ }^{1}$ Faculty of Engineering, Taif University, Taif, Saudi Arabia, \\ ${ }^{2}$ Computers and Systems Engineering Department, Faculty of Engineering, Al-Azher University, Egypt,
}

\begin{abstract}
This paper concentrates on the identification of Multiple Inputs Multiple Outputs (MIMO) system data with and without noise by means of a hybrid-learning rule, which combines the back propagation and the Least Mean Squared (LMS) to identify parameters. We construct a neuro fuzzy model structure, and generate the membership function from the measured data based on deterministic and soft computing. The four cases has been done. The first two cases for deterministic without and with noise have done. The second two cases for ANFIS without and with noise also have been done.. The MIMO system model is represented as a set of coupled input-output MISO models of the Takagi- Sugeno type. Neuro fuzzy model of the system structure is incorporated easily in the structure of the model. The simulation is used to implement a MIMO spacecraft system using Matlab for moment_yaw, moment_pitch, and moment_roll as input, and velocity in inertial axis as output. Experimental results are given to show the effectiveness of this Adaptive Neuro Fuzzy System (ANFIS) model.

This paper attempts to present ANFIS control design framework for a class of nonlinear multiple-input multiple-output (MIMO) system with uncertainties. This research effort is motivated by the following considerations: (a) An appropriate reference model that accurately represents the desired system dynamics is usually assumed to exist and to be available, and yet in reality this is not the case often times; (b) In real world applications, there are many cases where controls are constrained within a physically allowable range, which presents another layer of difficulties to directly apply the reference model based inverse control; (c) It is difficult to consider optimal control even for the reference model as in general the analytic solution to the optimal control problem is not available. The simulation study has been focused on the identification of Multiple Input, Single Output (MISO) non-linear complex systems.
\end{abstract}

Index Terms: - Real Time workshop, Neuro Fuzzy Controllers, Adaptive Neuro Fuzzy Inference System (ANFIS)

\section{INTRODUCTION}

Neuro Fuzzy modelling and identification from measured data are effective tools for the approximation of uncertain nonlinear systems. So far, most attention has been devoted to single input single output (SISO) or multiple input single output, (MISO) systems. Recently, also methods to deal with multiple-input, multiple-output (MIMO) systems have been proposed [1], [2], [3]. The purpose of this paper is four-fold: First, it is to create the ANFIS model structure and the membership function for neuro fuzzy system from the measured data. Second, it is shown that the identification technique based on a hybrid-learning rule, which combines back- propagation and the least mean squared to identify parameters for MISO systems, can be extended to a MIMO system in a straightforward way. Third, a Matlab toolbox is used which implements the presented techniques. Four, an illustrative example of a three-input, three-output nonlinear dynamic process of spacecraft is given. It is shown that the neuro fuzzy identification approach leads to a significant improvement in comparison with standard measured data from the system. While modern control methods were becoming widespread, in addition to demanded repeatability and accuracy specifications, reliability and detection and isolation of probable faults have become an obligation for automatic control systems. In the early 70's, first studies were appeared on this subject. While the first studies on fault detection and isolation (FDI) were implemented for supervisory of chemical processes, following studies were extended to systems like air and spacecraft's, automobiles, nuclear reactors, turbines and HVACs with high reliability mandatories after especially aircraft accidents with high mortality. In 1991, with extending and increasing studies, IFAC SAFEPROCESS committee was founded and in 1993, this committee issued some definitions about fault types, fault detection and isolation, fault diagnosis and fault tolerant control (FTC) (Isermann \& Ballé, 1997). Robots are accepted as an assistant subsystem or an individual part of a complex system in most applications. In addition to applications like serial product lines in which they can work harder, faster and with higher accuracy than humans, they are assigned to missions like waste treatment in nuclear reactors, data and sample collection, maintenance in space and underwater tasks which can be very risky for humans. As a consequence, a fault in one product line may cause a pause in all connected lines even in flexible automation systems or a developing and undetected fault may cause abortion of a whole space or underwater 
mission with big money costs, it may even cause harm to humans. With the increase of these events in real-life applications and with 90 's, studies on robot reliability and fault detection and diagnosis in robotics have become common. In addition to these studies, NASA and US Army issued some standards on robots and on the reliability and fault possibilities of robot parts (Cavallaro \& Walker, 1994). This study is focused on model-based FDI schemes, how they can be applied to robot manipulators, how soft computing techniques can be used in these schemes and three different FDI schemes are proposed. Soft computing techniques which can overcome the difficulties of schemes using analytical methods for nonlinear systems are used as modelling. In this study, these tools are used directly and without any modification to give appropriate outputs for appropriate inputs. From this point of view, this study can be accepted as a bridge between model-based FDI and data- based FDI methods. Furthermore, in this study, in addtion to soft computing tools, a hybrid soft computing tool M-ANFIS (multiple-ANFIS) which combines and utilizes benefits of neural network (NN) and fuzzy logic (FL) is used for modelling and function approximation. The two-link robot manipulator used in the simulations can be seen simple but accepted as a test platform for most studies. Besides, it has sufficient specifications for implementation of newly proposed schemes.

The arrangement of this paper is as follows. In section 2, Benefits and Structure of Neuro-Fuzzy Modelling are given. Section 3 discusses the levels of identification. In section 4 the representation of Neuro Fuzzy Identification for Spacecraft is introduced. To study the performance of the proposed ANFIS for spacecraft system a set of experiments has been conducted the simulation model described and investigated in section 4 . Implementation ANFIS model is introduced in section 5. Section 6 introduces the conclusion.

\section{LITERATURE OVERVIEW}

Studies and methods on fault detection and isolation can be divided into two main groups: model-based methods and data-based methods (Chen \& Patton, 1999). Model-based methods are based on modelling the system and processing the difference signals between the model and the real system named as residuals. Data-based methods are based on processing the input and output signals of the system. Proposed schemes in this study are based on model-based FDI methods and studies on model-based FDI are examined in details. Information and surveys about data-based methods can be found in (Chen \& Patton, 1999; Patton et al., 2000a; Venkatasubramanian et al., 2003). Model-based fault diagnosis (detection and isolation) is defined as detection, isolation and characterization of faults in components of a system from the comparison of the system's available measurements, with a priori information presented by the system's mathematical model (Chen \& Patton, 1999). According to this definition, model-based FDI methods are formed of two steps. The first step is generation of difference signals called residuals between real and predicted or estimated output signals of the system. Discordance of these real and predicted or estimated output signals which means nonzero residual signals indicates a potential fault in the system. The second step is isolation of faults using these obtained difference signals according to a decision set. Model-based FDI methods can be classified according to the method used for residual generation and the decision set is defined according to the specifications of each residual generation method. The point of classification can not only be the method but also linearity (linear-nonlinear-bilinear) type of the system that will be dealt with. The methods used for linear systems can be classified into three main titles. The first and mostly used methods are observer based methods. The main idea behind these methods is to estimate the output of the system using the measurements with Luenberger observes and deterministic adjustments or with Kalman filters and stochastic adjustments (Frank \& Ding, 1997). Parity vector (relation) methods use definition of parallel or temporal redundancy which is named as defining a variable with two or more definitions and which can be obtained from measurements or analytical relations (Chow \& Willsky, 1984). Parameter estimation methods are based on the principle that accepts sudden changes in parameters like friction, mass, viscosity, resistance etc. using system identification methods as a sign of faults (Isermann \& Ballé, 1997; Moseler \& Isermann, 2000). In addition to proposing new methods, all these methods are investigated for the robustness against disturbances and uncertainties. A detailed survey about these methods and studies can be found in (Chen \& Patton, 1999; Patton et al., 2000; Frank \& Ding, 1997). Generally, two main approaches are adopted for FDI for nonlinear systems (Chen \& Patton, 1999). First approach linearizes nonlinear models around one or multiple equilibrium(working) points and generates residuals insensitive to parameter changes in small equilibrium point neighborhoods using robust techniques. This approach may give good and sufficient results only for systems with low level nonlinearities. But this approach is not suitable for nonlinear systems having highly nonlinear terms and wide working points. Second approach, as proposed for solving this problem, uses multiple nonlinear models for each working points. But this approach brings multiple FDI systems for each working points and this will be not practical for real-time implementations. As mentioned below, to solve these problems, FDI methods which can deal with nonlinear systems directly must be proposed and developed. Therefore, most of the methods proposed for linear systems are adapted for nonlinear systems. One of the approaches is to use analytical or deterministic nonlinear observers (Chen \& Patton, 1999; Patton et al. 2000; Frank \& Ding, 1997; Adjallah et al.,1994; Garcia \& Frank, 1997). Model of nonlinear systems is accepted as below: Model-Based FDI Schemes For Robot Manipulators Using Soft 
Computing Techniques 131 manipulators is given. Soft computing tools used in these schemes are introduced; furthermore, how they work and what their duties are in these schemes are explained in details. Most studies in the literature are interested in sensor faults and locked and free-swinging joint (actuator) faults. This study is interested in abrupt partial actuator faults defined to contribute model-based FDI studies for robot manipulators. Furthermore, most studies using soft computing techniques for FDI are interested in how the parameters in their soft computing tools can be defined in terms of faults and how they can be updated according to faults like adaptive learning. In this study, these tools are used directly and without any modification to give appropriate outputs for appropriate inputs. From this point of view, this study can be accepted as a bridge between model-based FDI and data- based FDI methods. Furthermore, in this study, in addition to soft computing tools, a hybrid soft computing tool M-ANFIS (multiple-ANFIS) which combines and utilizes benefits of neural network (NN) and fuzzy logic (FL) is used for modelling and function approximation. The two-link robot manipulator used in the simulations can be seen simple but accepted as a test platform for most studies. Besides, it has sufficient specifications for implementation of newly proposed schemes.

\section{STRUCTURE OF NEURO- FUZZY MODELLING.}

There are two primary advantages to the use of a neuro fuzzy logic-based modelling scheme when compared to conventional arithmetic methods [4]: Neuro fuzzy logic reduces the difficulties of modelling and analysis of complex systems. \# Neuro fuzzy logic is suitable for incorporating the qualitative aspects of human experience or the input output data of process.

An TSK Neuro fuzzy model is a computationally efficient platform that is well suited for implementation of nonlinear associations through the construction of many piecewise linear relationships [5]. The need to approximate such a function is obvious given the nonlinear nature of Spacecraft. The proposed architecture is referred to as ANFIS, standing for adaptive-network- based Fuzzy Inference System. We describe how to decompose the parameter set in order to apply the hybrid- learning rule.

ANFIS architecture for simplicity, we assume the fuzzy inference system under consideration has two inputs $x$ and y and one output z. Suppose that the rule base contains two fuzzy if-then rules of Takagi and Sugeno's type [6]:
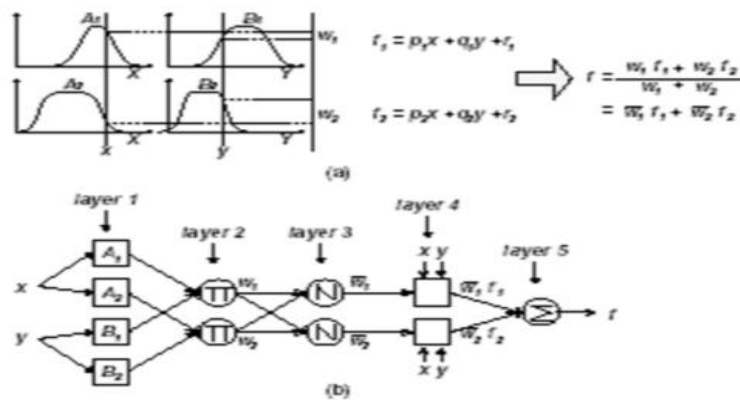

Fig (1)-a) Type-3 fuzzy reasoning; (b) Equivalent ANFIS (type-3 ANFIS)

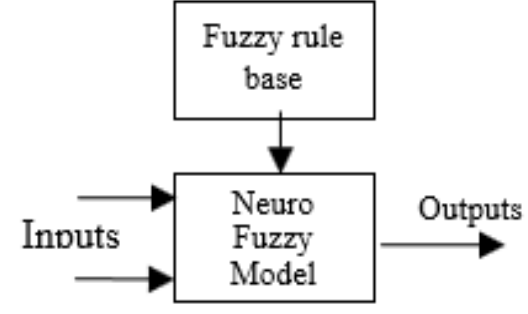

Fig. (2) general structure of an TSK Neuro fuzzy model

Rule 1: If $x$ is $A 1$ and $y$ is $B 1$, then $f 1=p 1 x+q 1 y+r 1$,

Rule 2: If $x$ is $A 2$ and $y$ is $B 2$, then $f 2=p 2 x+q 2 y+r 2$,

Then the type -3 fuzzy reasoning is illustrated in Fig (1.a), and the corresponding equivalent ANFIS architecture (type-3 ANFIS) is shown in Fig (1.b). The node functions in the same layer are of the same function family as described below:

Layer 1: Every node $\mathrm{i}$ in this layer is a square node with a node function

$O_{i}^{1}=\mu A_{i}(x)$,

Where $\mathrm{x}$ is the input to node $\mathrm{i}$, and $\mathrm{A} i$ is the linguistic label (small, large, etc.) associated with this node function. In other words, is the membership function of $\mathrm{A}_{1} \mathrm{O}^{\mathrm{i}}{ }_{\mathrm{i}}$ and it specifies the degree to which the given $\mathrm{x}$ satisfies the quantifier $\mathrm{A}_{i}$. Usually we choose ( ) A x i $\mu$ to be bell-shaped with maximum equal to 1 and minimum equal to 0 , such as 


$$
\mu_{A i}(x)=\frac{1}{\left[\left(\frac{x-c_{i}}{a_{i}}\right)^{2}\right]^{b_{t}}},
$$

OR

$$
\mu_{A i}(x)=\exp \left\{-\left[\left(\frac{x-c_{i}}{a_{i}}\right)^{2}\right]^{b_{i}}\right\}
$$

Where $\{a i$, bi, ci $\}$ is the parameter set. As the values of these parameters change, the bell shaped functions vary accordingly, thus exhibiting various forms of membership functions on linguistic label Ai. In fact, any continuous and piecewise differentiable functions, such as commonly used trapezoidal or triangular-shaped membership functions, are also qualified candidates for node functions in this layer. Parameters in this layer are referred as premise parameters.

Layer 2: Every node in this layer is a circle node labelled $\Pi$ as shown in Fig. (1), which multiplies the incoming, signals and sends the product out. For instance,

$$
\omega_{i}=\mu_{A i}(x) \times \mu_{B i(y)} i=1,2 \text {. }
$$

Each node output represents the firing strength of a rule. (In fact, other T-norm operator that performs generalized AND can be used as the node function in this layer).

Layer 3: Every node in this layer is a circle node labelled N. the i-th node calculates the ratio of the i-th rule's firing strength to the sum of all rules' firing strengths:

$$
\boldsymbol{\omega}_{i}=\frac{\omega_{i}}{\omega_{1}+\omega_{2}}, \quad i=1,2 \ldots . \mathrm{n}
$$

For convenience, outputs of this layer will be called normalized firing strengths.

Layer 4: Every node $\mathrm{i}$ in this layer is a square node with a node function

$$
o_{i}^{4}=\varpi_{i} f_{i}=\varpi_{i}\left(p_{i} x+q_{i} y+r_{i}\right) \text {, }
$$

Where $\varpi_{i}$ is the output of layer 3, and is the parameter set. Parameters in this layer will be referred to as consequent parameters. $\mathrm{p}_{i}, \mathrm{q}_{i}, \mathrm{r}_{i}$

Layer 5: The single node in this layer is a circle node labelled $\sum$ that computes the overall output as the summation of all incoming signals,

$$
o_{1}^{5}=\text { overalbutput } \sum_{i} \omega_{i} f_{i}=\frac{\sum \omega_{i} f_{i}}{\sum \omega_{i}}
$$

Thus we have constructed an adaptive network, which is functionally equivalent to a type-3 fuzzy inference system. The general structure of an TSK Neuro fuzzy model with $r$ inputs $x_{1}, x_{2}, \ldots x_{r}$ and one output $y$ is represented graphically in Fig (2).

\section{LEVELS OF IDENTIFICATION}

Identification of the neuro fuzzy model is considered to be calculating procedure where the parameters in the fuzzy rule base and/or in the data-base are to be determined in such a way so as to minimize a given performance index [7].

The general scheme of the identification procedure is shown in Fig (3). The identification process in which the neuro fuzzy model is created by a numerical iterative procedure for updating the membership parameters and weights of the model will be further called Learning of the Neuro Fuzzy Model [7]. According to the structure of the fuzzy model in Fig (3). There could be three different levels of identification, where different parts of the model are adjusted (separately or simultaneously), as follows:

fuzzy model in Fig (3). There could be three different levels of identification, where different parts of the model are adjusted (separately or simultaneously), as follows: 


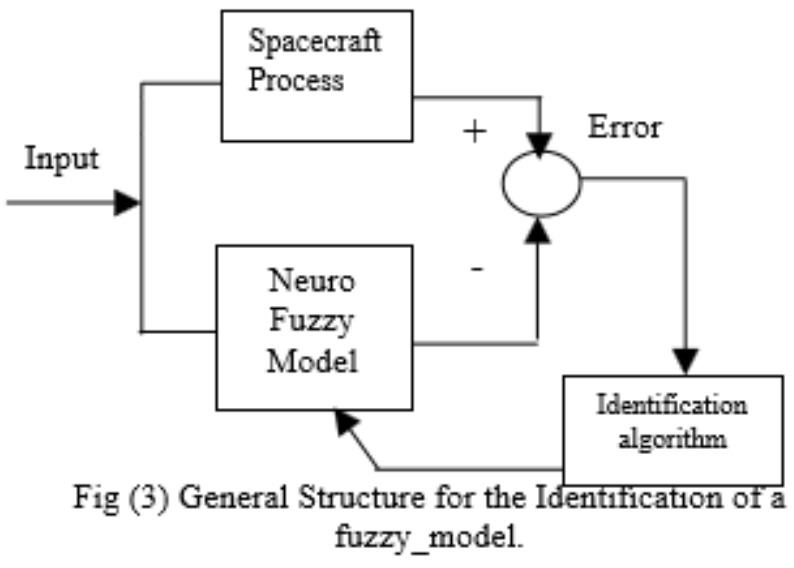

\section{III.I Structure Identification}

The objective here is to determine the linguistic structure of the fuzzy model, namely the fuzzy rule base [8]. It has the following components: the number of linguistic variables for each separate input; and the number of fuzzy rules as well as the content (completeness) of each rule, i.e. the number of inputs (linguistic variables) taking part in this rule.

The structure identification is directly related to the linguistic knowledge of the process behavior. If a sufficient and true linguistic knowledge exists, then the structure identification can be omitted as a procedure since the linguistic structure of the model is already known [9].

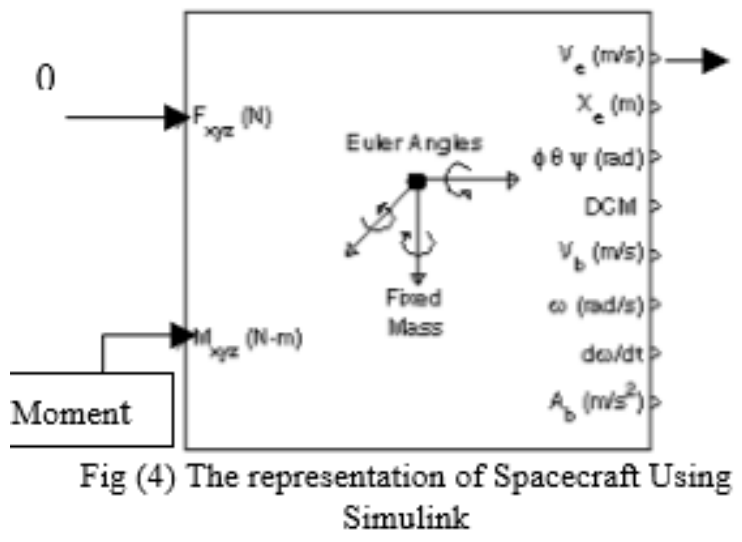

\subsection{Antecedent Parameters Identification}

This is the next (lower) level of the overall identification procedure, where the parameters of the set a in the database Fig (3) have to be adjusted in order to minimize a given performance index [10]. From a physical viewpoint this means that the parameters of the membership functions that define their shape and location have to be tuned so as to best fit the linguistic representation of the process. This level of identification can also be considered as a fine-tuning of the structure identification from the upper level 1.

\subsection{Consequent Parameters Identification}

This is the bottom level of identification related only to the algebraic output part of the fuzzy model [11]. In other words, this is a calculation procedure, which assumes that the linguistic parts of the model, namely the fuzzy rules and membership functions' locations and shapes, have already been defined.

\section{NEURO FUZZY IDENTIFICATION FOR SPACECRAFT}

The Inputs and Outputs data for Spacecraft Using Simulink are shown in Fig (4). The first input to the block is a vector containing the three applied forces (Fxyz). The second input is a vector containing the three applied moments (Mxyz). The first output is a three-element vector containing the velocity in the Earth-fixed reference frame. The second output is a three-element vector containing the position in the Earth-fixed reference frame. The third output is a three-element vector containing the Euler rotation angles [roll, pitch, yaw], in radians. The fourth output is a 3-by-3 matrix for the coordinate transformation from Earth-fixed axes to bodyfixed axes. The fifth output is a three-element vector containing the velocity in the body-fixed frame. The sixth 
output is a three-element vector containing the angular rates in body-fixed axes, in radians per second. The seventh output is a three-element vector containing the angular accelerations in body-fixed axes, in radians per second squares. The eighth output is a three-element vector containing the accelerations in body-fixed axes [12]. In this study, inputs and outputs of interest are (momentYaw, momentPitch, momentRoll), and outputs are velocities only, this will be enough and have practical importance. The inputs and outputs are applied to our system are shown in Fig (5) and Fig (6)

A. Data Collection. Data for training, testing and checking are obtained from the 6-degree of freedom given by Matlab toolbox. The training data contains 130 samples, 120 samples for testing data and 50 for checking data.

B. Training of the Model ANFIS is used to create a neuro fuzzy inference system (NFIS) that emulates behaviour of the spacecraft system. Before training can occur, an initial NFIS is created [13]. In this case, a blind NFIS is used, i.e. the initial NFIS contains knowledge of the target behaviour from measured data. With training, testing, and checking data established, the inference system is designed with three inputs (momentyaw, momentpitch, momentroll), and three output velocity in inertial axis (Vo1, Vo2, Vo3). It is assumed that there is no time delay between the input and output.

The following steps outline the development of the neuro fuzzy model: 1) Collect ample training, testing and checking data as produced by the target model. 2) Set initial membership function for input and output before training
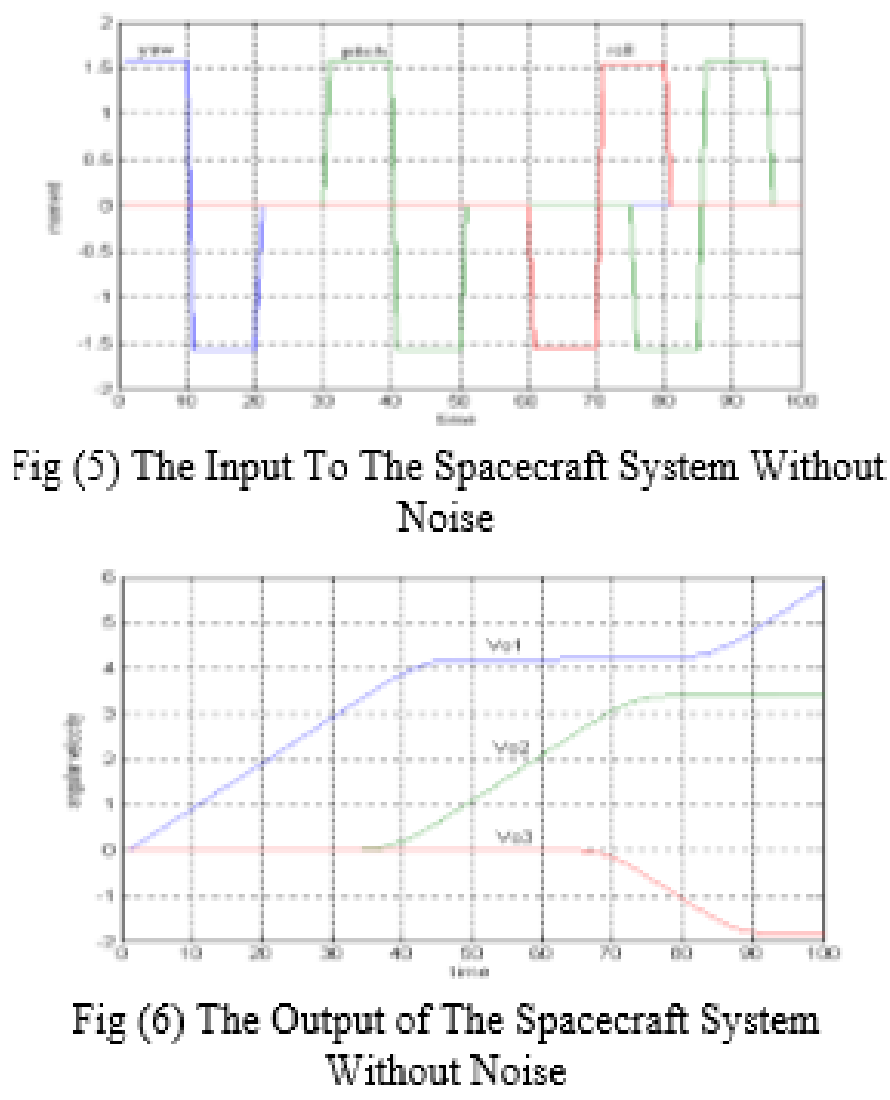

3) Use ANFIS to create a neuro fuzzy model that relates (momentyaw, momentpitch, momentroll) as inputs and velocities in inertial axis as output of the Spacecraft. 4) Set no. Of training data, testing data, checking data and choose no. Of epoch $=500$ and start training. If testing data is acceptable then checking other data else increase number of epoch. 5) If checking data is acceptable then select model and go to implementation else change the shape of membership function, go to step 2, and repeat these steps until satisfaction. 6) Validate the new model through comparison of its output of ANFIS model to the output of the target model, given the same identical inputs. To study the performance of the proposed ANFIS for spacecraft system, a set of experiments has been conducted the simulation model described in this section. Each experiment represents the performance of the spacecraft system under different operation conditions. We present here four different cases relevant to the system analysis. The following analysis tree schematics of these four cases are shown in Fig (7). 


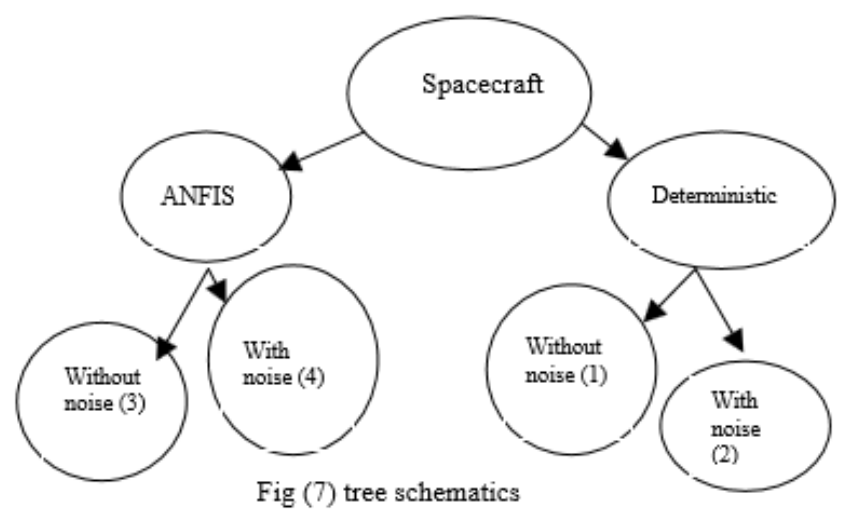

The following types of graphs present the input- output of the spacecraft, ANFIS system in each case: 1-the graph of the input momentyaw, momentpitch, momentroll 2-the graph of the output velocities (Vo1-Vo2-Vo3) To assess the performance of the process in each case. The first case is considered as the reference case without noise and the third case are compared with respect to it. The second case is considered as the reference case which taking noise into consideration and the fourth case is compared with respect to it. The sequence of steps, which uses in the implementation, is shown in Fig (8). The following three types of performances indices can be used for comparison:

1-the error through training. 2- the graph of average percentage errors from equation. 3-the error between ANFIS model and process output.

Case (1): We generate the multiple input-output data of spacecraft system, which not talk's noise into consideration. The inputs to our system are momentyaw, momentpitch, momentroll, the output of the system is velocity (Vo1-Vo2-Vo3). Programming or

Simulink under Matlab can implement this Simulation for spacecraft system. Fig (9) shows the input-output data model using ANFIS.

Case (2): We generate the multiple input-output data of spacecraft system taking noise into consideration as shown in Fig (10). The top left of Fig (15) show the input to the spacecraft system after adding noise and the top right show the first output of the velocity of spacecraft system.

Case 3: ANFIS (without noise) This Case presents the simulation results for a spacecraft of the proposed type3 ANFIS with pattern on line learning. In this application, ANFIS is to model nonlinear functions and the results are compared with the output of the spacecraft system. In conventional fuzzy inference system, an expert who is familiar with the system to be modelled decides the number of rules. In our simulation, however, no expert is available and examining the desired load input-output data chooses the number of membership functions assigned to each input variable. After the number of membership functions associated with each input is fixed, the initial values of the premise parameters are set in such a way that the membership functions are equally spaced along the operating range of each input variable. After that, we generate the structure of ANFIS model structure and determine the number of epochs, tolerance, and optimization method (hybrid between back propagation and least squares. After that, we start the training to generate the ANFIS model structure, membership function, and rule base. 


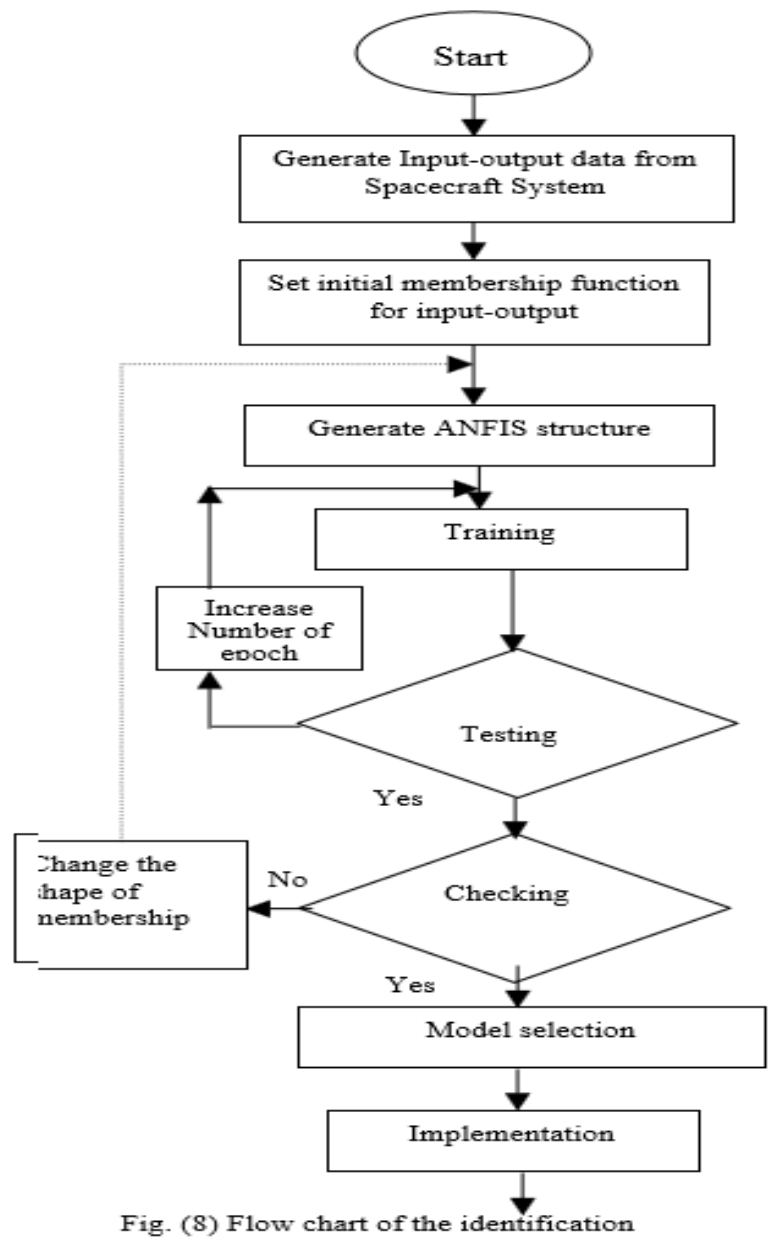

From simulation, we can show the results. Fig (11) shows The ANFIS Model Structure of MIMO for Spacecraft Using Matlab. The ANFIS used here contain 27 rules, with 3 membership functions being assigned to each input variable. Initial and final input-output membership functions before and after training are introduced in Fig (12a.b). The training data contains 130 samples for training data, 120 samples for testing data and 50 for checking data. The training data was used for the training of ANFIS, while the checking data was used for verifying the identified ANFIS only. To compute the average percentage error we used the following equation [14]. Average Percentage Error (APE)

$$
\mathrm{APE}=\frac{1}{p} \sum_{i=1}^{p} \frac{|\mathrm{T}(i)-\mathrm{O}(i)|}{|\mathrm{T}(i)|} * 100 \%
$$

Where $\mathrm{p}$ is the number of data pairs; T(i) and O(i) are i-th desired output and calculated output, respectively. Fig (13.a) shows the system Response of training data (Average percentage error $=0.42837$ through training). And, Fig (13.b) shows the system 

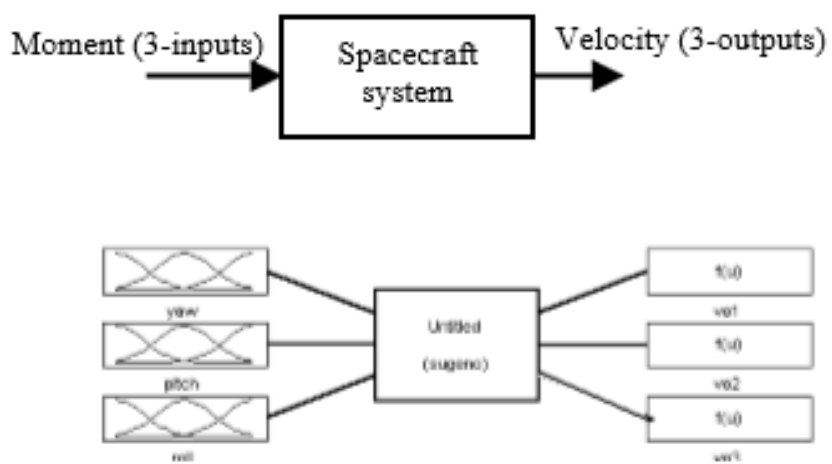

Fig (9) the input-output model using ANFIS

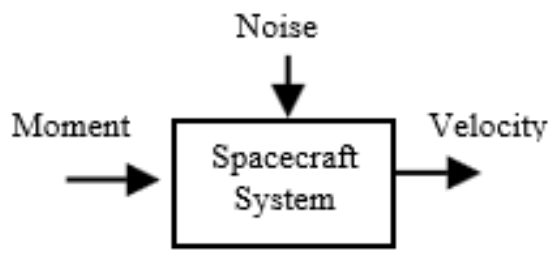

Fig (10) Spacecraft system after Adding Noise

Response of testing data (Average percentage error $=0.82837$ through testing). Fig (13.c) show the system response of checking data with Average percentage error=1.62837 through checking). Fig (13.d) shows the APE through training APE=0.4376. Fig (13.e) shows the system response for rule base after training Data for changing the input membership function. Fig (13-f) shows the system response of surface for input momentsyaw, momentspitch, and with one output Vo1.

Moment The samples of rules base generated in the simulation are: Velocity

If yaw is y1and Pitch is p1 and roll is $\mathrm{r} 1$ then (Vo1 is yprout1,Vo2 is yprout2,Vo3 is yprout3)

If yaw is y1and Pitch is p2 and roll is $\mathrm{r} 2$ then (Vo1 is yprout3,Vo2 is yprout2,Vo3 is yprout1)

If yaw is y3and Pitch is p1 and roll is $r 3$ then (Vo1 is yprout1,Vo2 is yprout3,Vo3 is yprout2) 


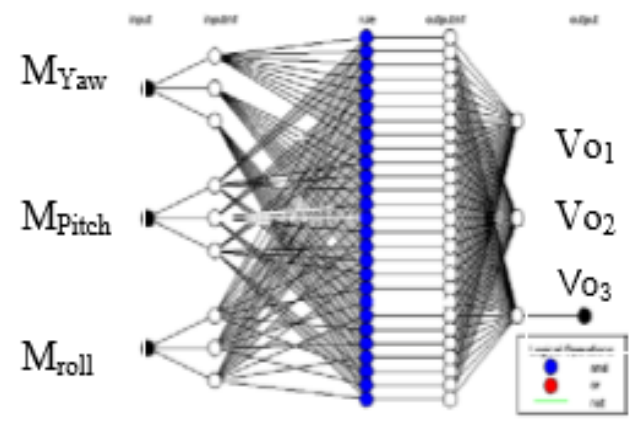

Fig (11) ANFIS Model Structure I

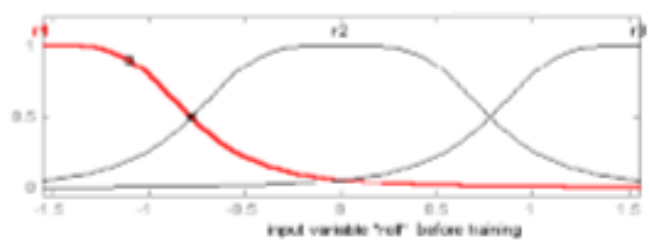

Fig (12) a. initial membership function for three inputs before training
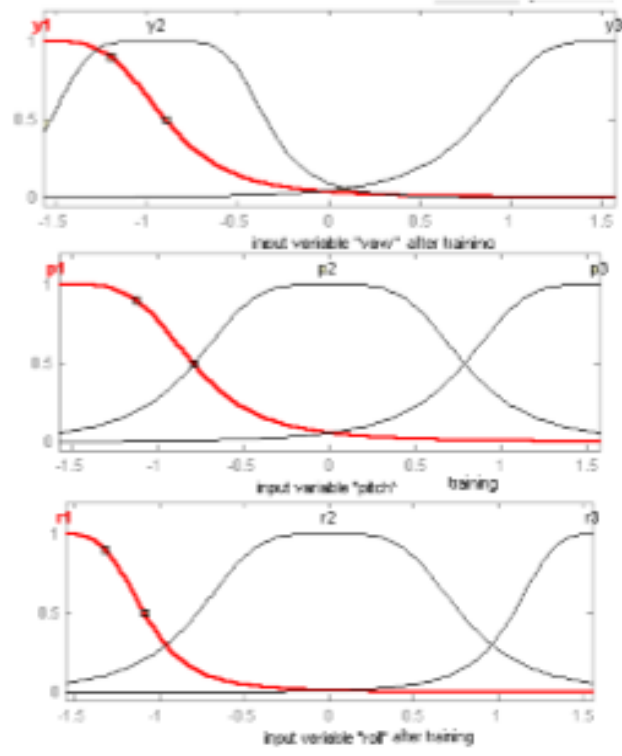

Fig (12) b. membership function after training

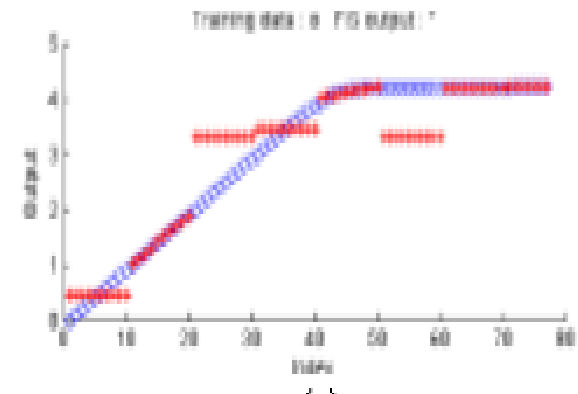

(a)

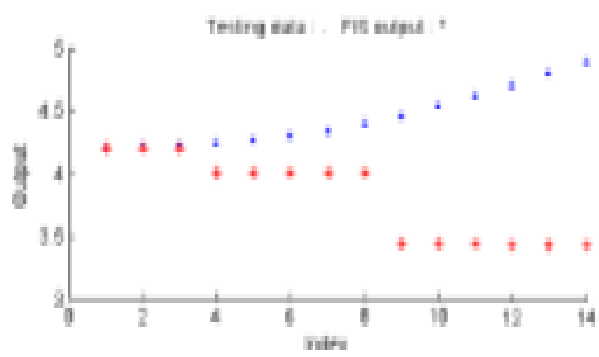

(b)

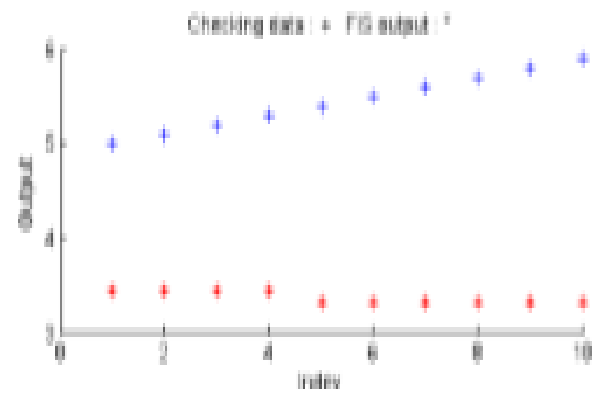

(c)

Fig (13) a-System Response of trairing data- (b). testing data - (c) System Response of testing 


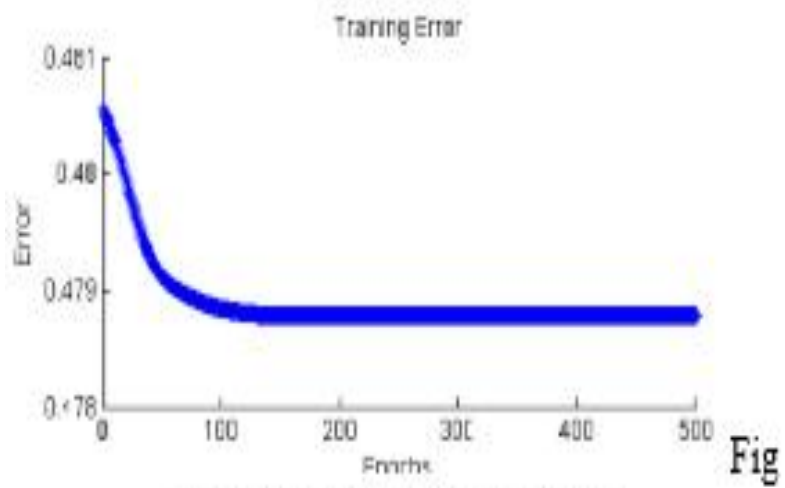

(13.d) APE Through training
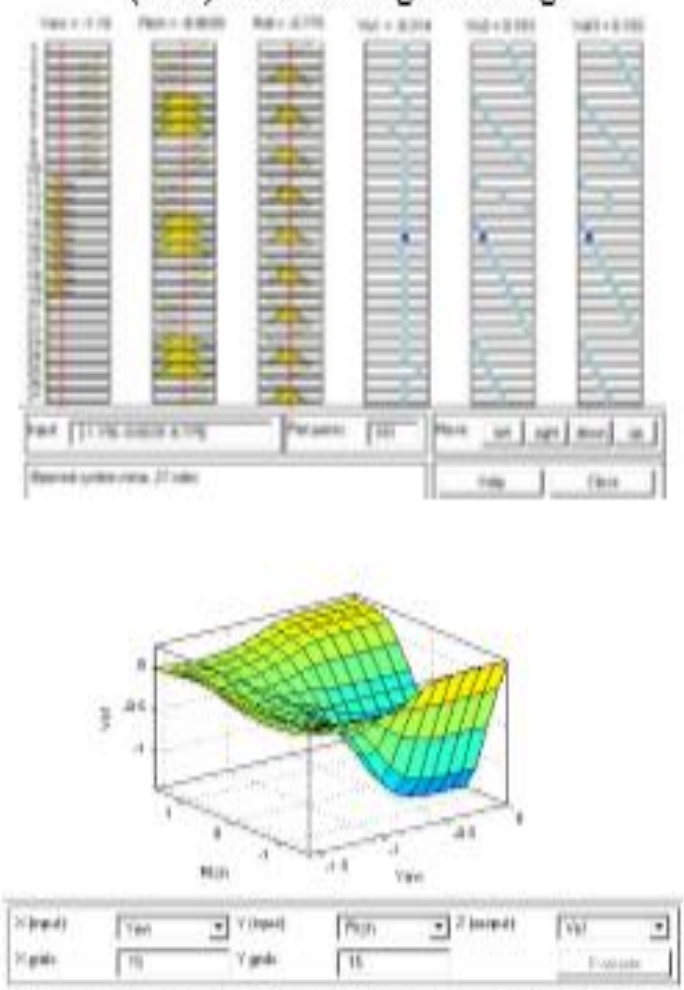

Fig (13.e) System Response for rule base after training Data for changing the input membership function ( $f$ ) System response of surface for input yaw, pitch, and with output $\mathrm{Vol}_{0}$ 

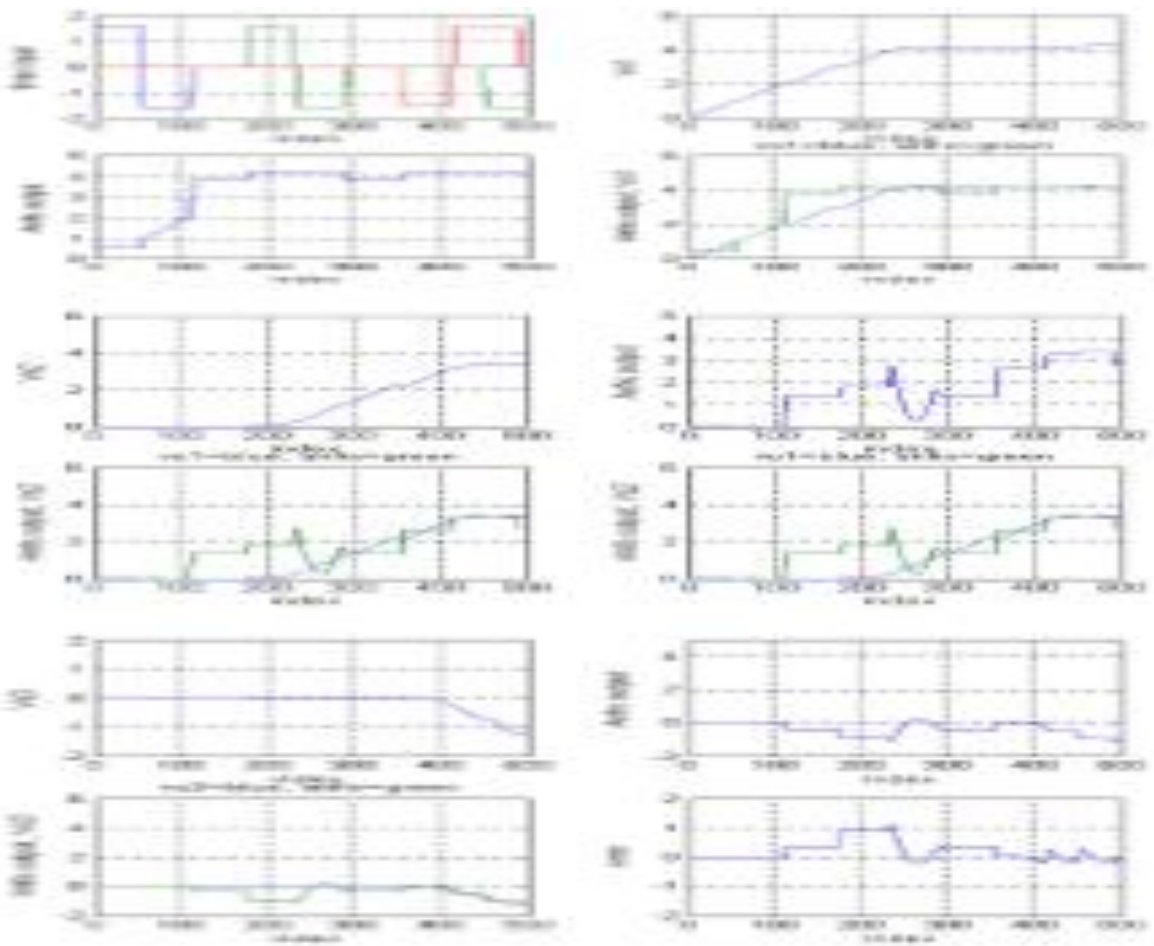

Time

Fig (14) Comparison between ANFIS Mode1 and Spacecraft system without noise
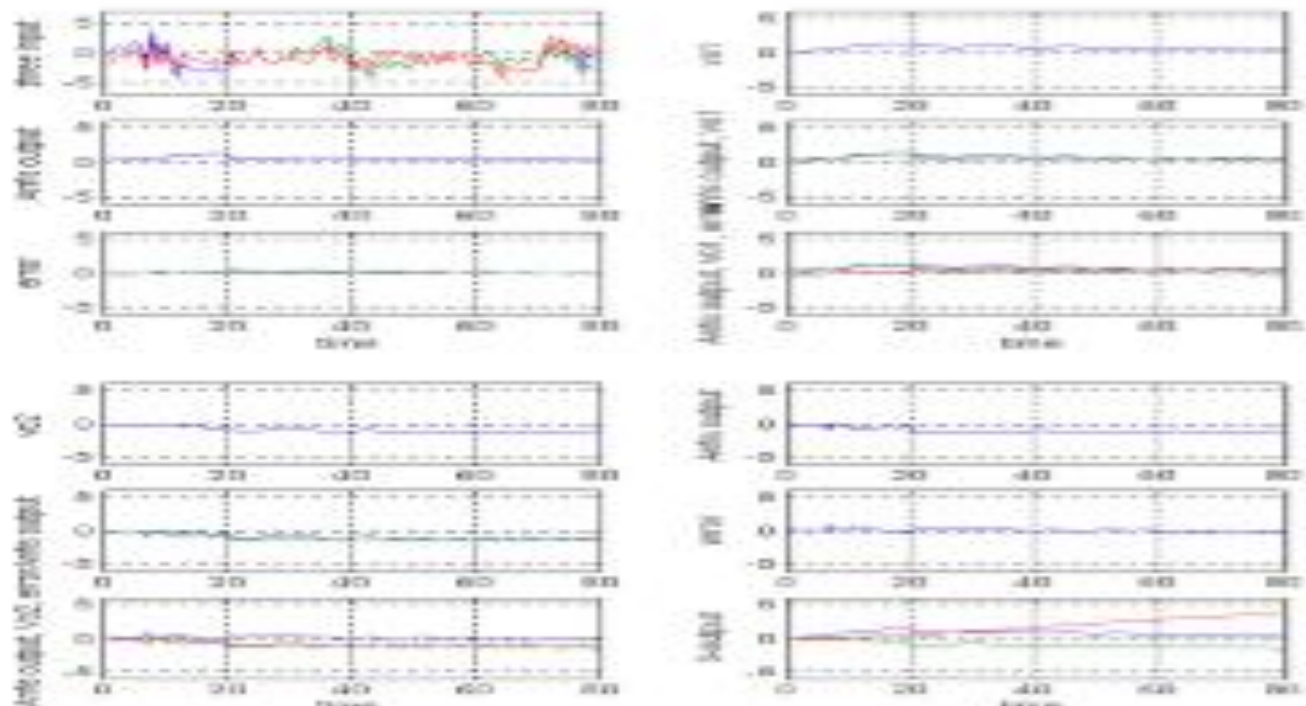

E
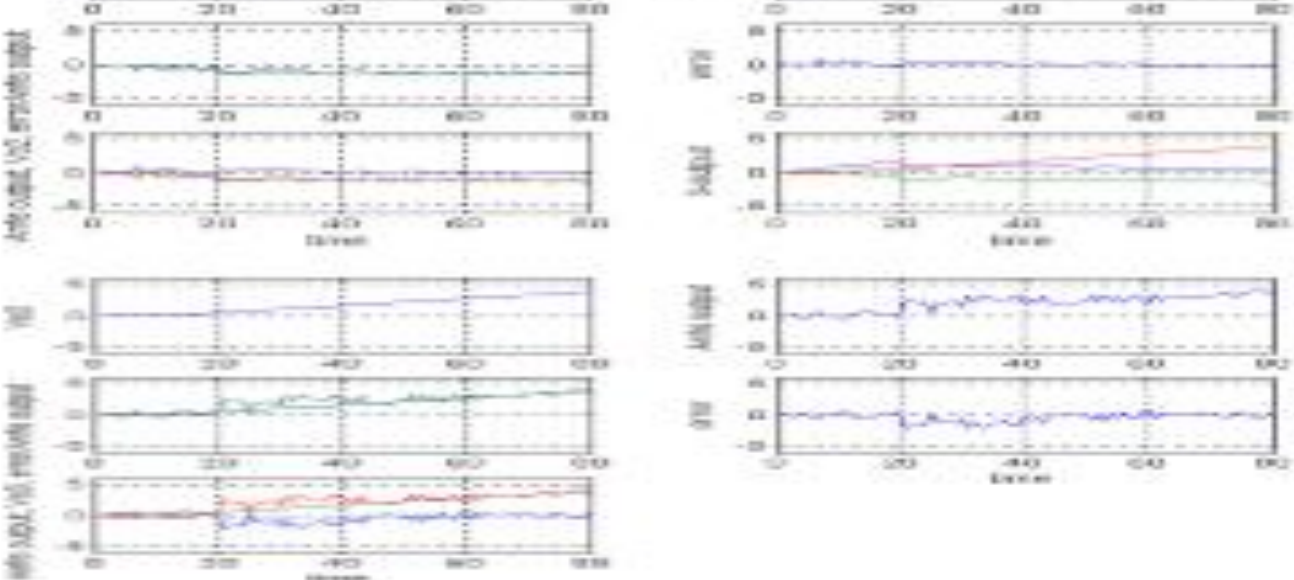

Fig (15) Comparison between ANFIS Mode1 and Spacecraft system with noise 
In this stage we can construct this ANFIS model. In the following section, we can implement the anfis model and compare the output of ANFIS with the output of spacecraft.

\section{IMPLEMENTATION}

After completing the training, testing, and checking system of case 3, we can implement the ANFIS model and compare the ANFIS model output with the output of spacecraft in each case. Fig (14) shows the comparison between ANFIS Model and Spacecraft system for the same input with the three outputs. We found the result of system identification with minimum error rate. Case 4 is the same of case 3 , but in case 4 we talk noise into consideration. Fig (15) shows the comparison between the result of ANFIS Model and Spacecraft system for the same input with the three outputs (velocity).

\section{CONCLUSIONS}

The four cases are implemented base on deterministic and soft computing. The gathering od data is commutated with and without noise. The ANFIS model structure based on measured data of spacecraft system are introduced. Rules are generated automatically and linguistic neuro fuzzy model is constructed. By employing a hybrid learning procedure, the proposed architecture can refine fuzzy if-then rules obtained from human expertise to describe input-output of a complex system. To generate inference mechanisms by the simulation process based on input-output TS fuzzy models was presented for MIMO dynamic process. The MIMO spacecraft is system identified using ANFIS under Matlab. If human expertise is not available we can still set up intuitively reasonable membership functions and start the learning process to generate a set of fuzzy if-then rules to approximate the desired data set as shown in the simulation. Simulation for spacecraft is introduced and implemented to illustrate the advantages of ANFIS model and also the software implementation in Matlab. Experimental results are introduced to show the effectiveness of this ANFIS model.

\section{REFERENCES}

[1] Tharwat O S Hanafy. "A New Algorithm to Model Highly Nonlinear System based Coactive Neuro Fuzzy Inference System." International Journal of Computer Applications 94(17):9-20, May 2014. Published by Foundation of Computer Science, New York.

[2] Tharwat O. S. Hanafy, Al-Osaimy, Mosleh M. Al-Harthi "Identification of Uncertain Nonlinear MIMO Spacecraft Systems Using Coactive Neuro Fuzzy Inference System (CANFIS)", International Journal of Control, Automation and Systems,PP.25-37, Pub. 2014.

[3] Tharwat O. S. Hanafy, Al-Osaimy "Facilitation Rule Base for Solidification of Nonlinear Real Plant System ", International Journal of Control, Automation and Systems, ISSN 2165 - 8277, PP.1-9, Pub. Date: 2014-01-15.

[4] Tharwat O. S. Hanafy, and Kamel A. Shoush. "Dynamic Evolving Neuro Fuzzy Systems of Qualitative Process " International Journal of Control, Automation and Systems, ISSN 2165 - 8277, PP.1-9, Pub. Date: 2014-01-15.

[5] Tharwat O. S. Hanafy, H. Zaini, Kamel A. Shoush "Recent Trends in Soft Computing Techniques for Solving Real Time Engineering Problems" International Journal of Control, Automation and Systems, ISSN 2165 - 8277 PP.27-33, Pub. Date: 2014.

[6] Tharwat O. S. Hanafy, and M. Kamel "Simplification of Rules Base for Inverted Pendulum using ANFIS" Indianan Journals Internationals, 2014

[7] Tharwat. O. Hanafy, "Adaptive Neuro Fuzzy Systems for Dynamic Qualitative Modeling Process" Nature and Sicence Journal ,V 8 , 2010

[8] Tharwat. O. Hanafy, "Design and Validation of Real Time Neuro Fuzzy Controller for Stablization of Pendulum Cart System" Life Sicence Journal ,V 8 ,I 1, 2011.

[9] Tharwat. O. Hanafy, "Neuro Fuzzy Modeling Scheme for the Prediction of Air Polution" Journal of American Science, V 6, 12, 2010.

[10] Tharwat. O. Hanafy, "A modified Algorithm to Model Highly Nonlinear systems " Journal of American Science, V 6, 12, 2010. 
[11] Tharwat. O. Hanafy, Tarek Soph, Awd K. "A systematic Algorithm to construct Neuro Fuzzy Inference Systems" $16^{\text {th }}$ International Conference in software Engineering and Data Engineering July 9-11-2007, ISCA.

[12] Tharwat. O. Hanafy, "Stablization of Inverted Pendulum System using Particular Swarm Optimization" the $8{ }^{\text {th }}$ International Conference on Informatics and systems INFOS 2012, 14-16 may.

[13] Tharwat. O. Hanafy, Emad Massamir, Osama amr, "Extracting Variables Size Secret Keys From Voice Key", New York Sicence Journal, V 4, 2011.

[14] Tharwat. O. Hanafy, Tarek Soph, Awd K. "Hybrid Learning Rules for Identifying Spacecraft system" 20 th International Conference on Computers and Their Applications, CATA 2005, Orleans, USA.

[15] Tharwat. O. Hanafy, Tarek Soph, Awd K. "Neuro Fuzzy Techniques using Matlab /Simulink Applied to Real Process" $14^{\text {th }}$ International Conference on Intelligence and Adaptive systems, and soft ware Engineering, IASSE, 2005.

[16] Tharwat. O. Hanafy," Recent Trends in Evolutionary Computations on Organic Mechanism Simulation for Control Systems", Global Advanced Research, technology and Innovation 2012

[17] Ruiyun Qi, Limeng Zhu and Bin Jiang," Fault-Tolerant Reconfigurable Control For MIMO Systems Using OnLine Fuzzy Identification ”, International Journal of Innovative Computing, Information and Control ICIC International 2013 ISSN 1349-4198Volume 9, Number 10, October 2013 pp. 3915-3928

[18] Wuhan, Hubei, China, "Modelling, Identification \& Control (ICMIC)", 2012 Proceedings of International Conference on June 2012,467 - 471 .

[19] A. Gegov, "Multilayer fuzzy control of multivariable systems by active decomposition". Int. Journal of intelligent systems, 12, 1997.

[20] Xiao-Jun Zeng, Madan G. Singh," Fuzzy Bounded Least Squares Method for Systems Identification" March 31 - April 02, 1996, University of Manchester Institute of Science and Technology

[21] Carmona, P. Castro, J.L. Zurita, J.M. "FRIWE: Fuzzy Rule Identification With Exceptions", IEEE Transactions on Fuzzy Systems, Publication Date: Feb. 2004 on page(s): 140- 151Volume: 12, Issue

[22] Yu, W. Li, X. "Fuzzy Identification Using Fuzzy Neural Networks With Stable Learning Algorithms", IEEE Transactions on Fuzzy Systems, June 2004 On page(s): 411-420 Volume: 12, Issue: 3, ISSN: 10636706.

[23] Jang R, Sun C T and Mizutani E, Neuro-fuzzy and soft computing: A computational Approach to Learning and Machine Intelligence, Prentice Hall NJ, 1997.

[24] Abraham A, A Soft Computing Approach for Fault Prediction of Electronic Systems, The 2nd International Conference on Computers in Industry ICCI 2000, Bahrain, pp. 83-91, November 2000

[25] Juang Chia Feng, Lin Chin Teng, an online self constructing Neural Fuzzy Inference Network and its Applications, IEEE Transactions on Fuzzy systems Vo16, No.1, pp. 12-32, 1998.

[26] Ho Jae Lee, Hagbae Kim, Young Hoon Joo, Wook Chang, Jin Bae Park "A new intelligent digital redesign for T-S fuzzy systems: global approach" IEEE Transactions on Fuzzy Systems", April 2004 On page(s): 274-284,Volume: 12,Issue: 2ISSN: 1063-6706

[27] Lin C T and Lee CSG, Neural Network based Fuzzy Logic Control and Decision System, IEEE Transactions on Computer. (40(12): pp. 1320-1991.

[28] Bherenji H R and Khedkar P, Learning and Tuning Fuzzy Logic Controllers through Reinforcements, IEEE Transactions on Neural Networks, Vo1 (3), pp. 724-740, 1992.

[29] Lin C T and George Lee C S Neural Fuzzy Systems: A Neuro-fuzzy Synergism to Intelligent Systems, prentice Hall NJ, 1995.

[30] J.S. R. Jang, "ANFIS: Adaptive-Network-Based Fuzzy Inference System," IEEE Trans. on Syst., Man, and Cybern. Vol. 23, no. 3, pp. 665-685, 1993.

[31] Amine Tabelsi, Frederic lafont "Identification of Nonlinear Multivariable Systems by Adaptive Fuzzy Takagi-Sugeno Model," International Journal of Computational Cognition, September 2004, page 137153.

[32] Nürnberger, A., Nauck, D., Kruse, R., Merz, L., A Neuro-Fuzzy Development Tool for Fuzzy Controllers under MATLAB/SIMULINK, In Proc. of the 5th European Congress on Intelligent Techniques \& Soft Computing (EUFIT '97), Aachen, Germany, 2012 
[33] Pechmann S, Frydman J, ". Evolutionary conservation of codon optimality reveals hidden signatures of cotranslational folding. Nat. Struct. Mol. Biol. 2013;20:237-243.

[34] Klass DM, Scheibe M, Butter F, Hogan GJ, Mann M, Brown PO, "Quantitative proteomic analysis reveals concurrent RNA-protein interactions and identifies new RNA-binding proteins in Saccharomyces cerevisiae. Genome Res. 2013; 23:1028-1038.

[35] I.M. Kouatli, “A simplified fuzzy multivariable structure in a manufacturing environment.", Journal of intelligent Manufacturing, 3:365-387,1994.

[36] Y.Z. Lu. Iie, and C.-W, "Fuzzy modeling and expert optimization control for industrial process". IEEE Trans. On Control Systems Technology, 5:2- 12, 1997.

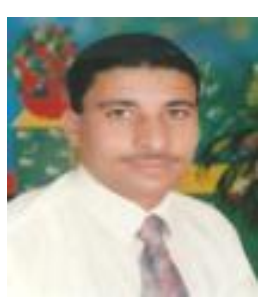

First author's

Dr Eng. Tharwat Owiss Hanafy received his doctoral degree in Computer engineering from Azhar University, Computers and System Eng. Dept. 2007. He is a lecturer in the Department of Computer engineering, Azhar University, Egypt. His research interests cover, Expert Systems, Fuzzy Systems, Neuro Fuzzy Systems, Neuro Fuzzy Controllers, Neuro Fuzzy Modeling, Adaptive Neuro Fuzzy Inference System (ANFIS), CANFIS,

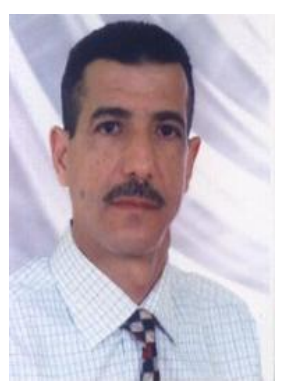

Nadjim MERABTINE received the B.Sc degree in Electrical and Electronics Engineering and the M.Sc degree in Microwave from Portsmouth Polytechnic Hants., United Kingdom, in 1978 and 1979, respectively. He received the M.Eng degree in electronics and D.Sc in Communication Engineering from Mentouri University, Constantine Algeria, in 1986 and 2003, respectively. He was a Graduate Engineer at Standard Telephones \& Cables Ltd., (STC), British Branch of ITT, Transmission Products Division, Basildon; Essex, United Kingdom from 1979 to 1980 .He was a Systems Development Engineer at PYE Telecommunication Ltd., Cambridge, United Kingdom from 1980 to 1981.He was a Lecturer at the National School of Civil Aviation \& Aeronautics, Constantine, Algeria from 1981 to 1983. He has been Assistant Professor, Associate Professor and Professor, respectively from 1983 to 2009 and Head of Research Team in the Electromagnetism \&Telecommunication Laboratory, at Mentouri University, Constantine, Algeria. He is currently Professor and Head of Electronics \& Communications Branch, Electrical Engineering Department at Taif University, Saudi Arabia, since 2009. He is Board Member Committee of The International Society of Management Engineers (ISME).His research interests are in the areas of electronics, communications engineering and cryptography.

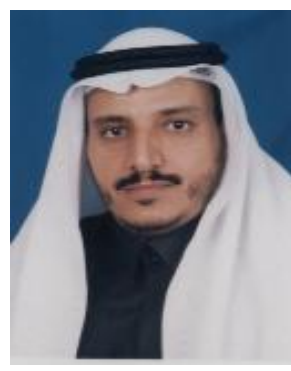

Mosleh M. Al-Harthi was born in Taif, Saudi Arabia, on October 15, 1966. He received the B.Sc. degree in electronics technology and engineering from Indiana State University, Terre Haute, USA, in 1996, and the M.S. degree in electronics technology and engineering from Indiana State University, Terre Haute, USA, in 1997. He received the Ph.D. degree in electrical engineering from Arkansas University, Fayetteville, USA, in 2001. He was an assistant professor at College of Technology in Jeddah, Saudi Arabia form 2001 till 2009. He is currently working as an associate professor at the Electrical Engineering Department, Taif University, Saudi Arabia. His research interests are in the areas of control engineering, electronics, and signal processing. 